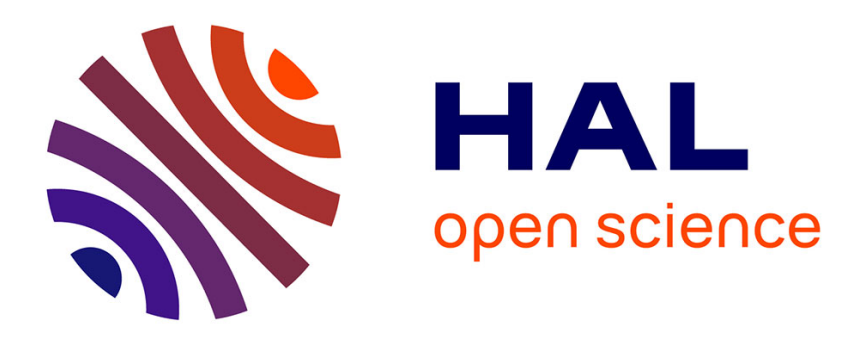

\title{
L'agriculture française dans la compétition mondiale Mélanie Gambino
}

\section{To cite this version:}

Mélanie Gambino. L'agriculture française dans la compétition mondiale. Outre-terre. Revue européenne de géopolitique, 2012, 33-34 (3), pp.307-320. 10.3917/oute.033.0307 . hal-01998282

\section{HAL Id: hal-01998282 \\ https://hal.science/hal-01998282}

Submitted on 30 Oct 2020

HAL is a multi-disciplinary open access archive for the deposit and dissemination of scientific research documents, whether they are published or not. The documents may come from teaching and research institutions in France or abroad, or from public or private research centers.
L'archive ouverte pluridisciplinaire HAL, est destinée au dépôt et à la diffusion de documents scientifiques de niveau recherche, publiés ou non, émanant des établissements d'enseignement et de recherche français ou étrangers, des laboratoires publics ou privés. 


\section{L'agriculture française dans la compétition mondiale}

Mélanie Gambino ${ }^{1}$

L'agriculture française se trouve de plus en plus intégrée aux flux d'échanges mondiaux de produits agricoles et alimentaires. Sur les marchés agricoles, elle doit faire face à un renversement des courants d'échanges qui a débuté il y a près de $15 \mathrm{ans}^{2}$. Les stratégies des grands pays agricoles visent à tirer parti au mieux de la réorientation du commerce mondial. Certains comme les États-Unis, le Brésil ou l'Argentine le font en misant sur l'exportation des commodities (matières premières). D'autres pays comme la Chine ou l'Inde (qui rassemblent $55 \%$ des agriculteurs de la planète) cherchent à renforcer leur souveraineté alimentaire, notamment dans le domaine des céréales $^{3}$. La France qui a occupé le rang de deuxième exportateur mondial jusqu'en 1995 perd progressivement sa place de grande puissance exportatrice. Elle n'est plus aujourd'hui que le quatrième exportateur mondial de produits agricoles, ex aequo avec le Brésil ${ }^{4}$. Toutefois, il convient de ne pas confondre l'affaiblissement progressif de la balance commerciale de l'agroalimentaire, marqué par la chute de l'excédent commercial agroalimentaire français ${ }^{5}$ avec un affaiblissement des ressources agricoles, du potentiel et de l'utilisation de la production. Et, dans cette compétition mondiale, la production agricole française, avec une valeur au prix de base de 65,8 milliards d'euros en 2010, est la première d'Europe. Elle contribue à concurrence de $18,6 \%$ à la production de l'UE à vingt-sept, suivie par l'Allemagne (12,7\%), l'Italie $(12,5 \%)$ et l'Espagne (11\%). Dans ce contexte où les conditions de la concurrence ont rapidement changé, quelle est donc la stratégie de la France? Comment se positionne-t-elle? Quels sont ses objectifs, ses capacités et son potentiel?

\section{Portrait et évolution de l'agriculture en France}

Depuis 1950, la tendance qui caractérise l'agriculture française est un accroissement de la production et de la productivité physique du travail agricole plus rapide que dans les autres secteurs de l'économie ${ }^{6}$. Une tendance à l'augmentation de la

\footnotetext{
Maître de conférences en Géographie rurale, UMR Dynamiques Rurales, Université de Toulouse 2 Le Mirail, gambino@univ-tlse2.fr

2 Demeter, 2010, rapport annuel.

3 Cf. Jean-Pierre Charvet, Atlas de l'agriculture. Comment pourra-t-on nourrir le monde en 2050?, Paris, Autrement, $2010,79 \mathrm{p}$.

4 Global Trade Atlas, 2012.

5 FAO, Rapport 2009.

6 Cf. Mélanie Gambino, Cécile Laisney, Julien Vert, Le monde agricole en tendance. Un portrait social prospectif des agriculteurs, Centre d'études et de prospective, SSP, ministère de I'Agriculture, de l'Alimentation, de la Pêche, de la Ruralité et de l'Aménagement du territoire, 2012.
} 
taille moyenne des exploitations et à la diminution de la Surface agricole utilisée (SAU) se poursuit aujourd'hui (voir figure $n^{\circ} 1$ ).

Figure $\mathrm{n}^{\circ} 1$ : Caractéristiques des exploitations agricoles françaises en 2010

\begin{tabular}{|c|c|c|c|c|}
\hline & \multicolumn{2}{|c|}{ Ensemble des exploitations } & \multicolumn{2}{|c|}{ dont moyennes et grandes exploitations } \\
\hline & 2010 & $\begin{array}{l}\text { Évolution depuis } 2000 \\
(\%)\end{array}$ & 2010 & $\begin{array}{l}\text { Évolution depuis } 2000 \\
(\%)\end{array}$ \\
\hline Exploitations agricoles (millier) & 490 & -26 & 312 & -19 \\
\hline $\begin{array}{l}\text { Chefs d'exploitation et coexploitants } \\
\text { (millier de personnes) }\end{array}$ & 604 & -21 & 423 & -13 \\
\hline $\begin{array}{l}\text { Salariés permanents hors famille } \\
\text { (millier de personnes) }\end{array}$ & 155 & -6 & 148 & -5 \\
\hline Travail agricole (millier d’UTA) & 751 & -22 & 651 & -20 \\
\hline Superficie agricole utlisée (millier d'hectares) & 26961 & -3 & 25096 & -2 \\
\hline Superficie agricole utilisée moyenne ${ }^{*}$ (hectare) & 55 & +31 & 80 & +22 \\
\hline Exploitations ayant des vaches laitières (millier) & 82 & -36 & 78 & -34 \\
\hline Cheptel de vaches laitières (millier de têtes) & 3717 & -11 & 3700 & -11 \\
\hline Troupeau moyen en vaches laitières (tête) & 45 & +38 & 48 & +36 \\
\hline Exploitations ayant des vaches nourrices (millier) & 121 & -27 & 88 & -21 \\
\hline Cheptel de vaches nourrices (millier de têtes) & 4100 & -5 & 3784 & -1 \\
\hline Troupeau moyen en vaches nourrices (tête) & 34 & +31 & 43 & +25 \\
\hline
\end{tabular}

* Superficie moyenne, y compris exploitations sans SAU.

Source : Aareste - Recensements aaricoles

Cette évolution s'appuie sur la mécanisation, les achats d'intrants, la spécialisation et l'agrandissement des exploitations, la répartition des activités avec la recherche (qui conçoit les itinéraires techniques) et avec les Industries agroalimentaires (IAA, pour vendre les produits agricoles bruts). Les systèmes de production sont transformés par l'adoption d'équipements toujours plus performants. Pour rentabiliser les investissements, les systèmes de production sont de plus en plus spécialisés (cf. figure $\mathrm{n}^{\circ} 2$ ) et visent l'accroissement de la productivité physique du travail. Cette tendance s'observe en grandes cultures: augmentation de la superficie par actif, simplification du travail, simplification et raccourcissement des rotations, augmentation du rendement par hectare. L'accroissement de la productivité du travail repose sur un capital fixe de plus en plus important par actif et sur un recours croissant aux consommations intermédiaires (herbicides surtout). Dans le système actuel, la fertilité du sol est davantage pilotée par les intrants que par les rotations (simplification des assolements). Dans les systèmes d'élevage bovin laitier, la même tendance s'observe: augmentation du nombre de vaches laitières par actif, augmentation de la production laitière par vache laitière, augmentation du nombre de vaches laitières par hectare et des unités fourragères par hectare. Comme pour les grandes cultures, l'accroissement de la productivité du travail repose sur un capital fixe de plus en plus important par actif et sur un recours croissant aux consommations intermédiaires au coût très élevé.

7 Cf. Marcel Mazoyer, Laurence Roudart, Histoire des agricultures du monde, Paris, Éditions du Seuil, 1997,705 p. 
Figure $\mathrm{n}^{\circ} 2$ : les bassins de production en France

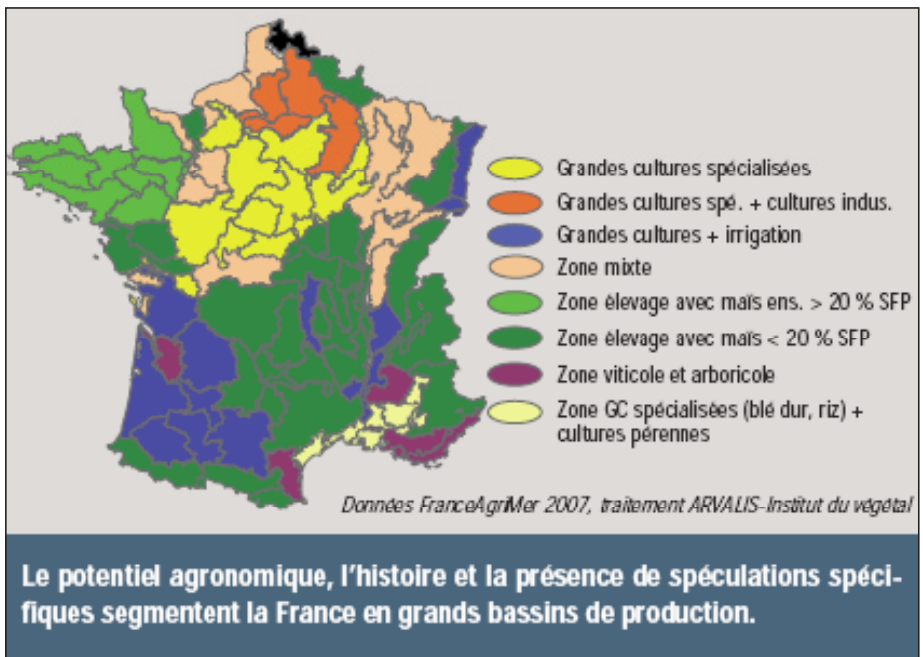

D’après Yannick Carel, Martine Julien, Valérie Leveau, «Exploitations céréalières françaises : quelle restructuration?», Perspectives agricoles, ${ }^{\circ} 372$, novembre 2010

Le dernier recensement agricole ${ }^{8}$ a dénombré 490000 exploitations et un million de personnes impliquées de façon régulière dans leur activité. Le nombre d'exploitation ne cesse de se réduire alors que la surface moyenne augmente avec 55 hectares. Pour compléter ce succinct panorama, rappelons que la profession agricole n'occupe plus aujourd'hui que $3,3 \%$ de la population active totale (contre $5,3 \%$ pour l'ensemble de l'Union européenne) et contribue à hauteur de 1,4\% du PIB. Ces chiffres peuvent paraitre dérisoires, surtout quand on se souvient que l'agriculture pesait 13,7\% du PIB en 1955. La statistique agricole en France n'est pas avare de données et peut parfois donner le tournis, mais nous en resterons là pour décrire la «ferme France». Il s'agit maintenant de voir comment un pays qui ne mesure que $550000 \mathrm{~km}^{2}$ et qui n'a qu'un million d'agriculteurs se positionne sur les marchés et dans la compétition mondiale.

\section{Queloues indicateurs sur les performances des productions ET DES EXPORTATIONS}

Le tableau ci-dessous souligne la place prépondérante de la France au sein de l'UE et met en avant une diversité de productions qui lui permettent se classer parmi les grands pays agricoles. En 2009, la France reste premier producteur européen de blé,

8 Agreste (service statistique de ministère de l'Agriculture, de l'Alimentation, de la Pêche et des Affaires rurales), 2010. 
soit le tiers du volume communautaire, de maïs malgré la concurrence croissante des nouveaux états membre (cf. figure $\mathrm{n}^{\circ} 3$ et voir en annexe pour un classement détaillé en quantité et en valeur).

Figure $\mathrm{n}^{\circ} 3$ : La place de la France dans les productions agricoles mondiales.

\begin{tabular}{|l|c|c|c|}
\hline & $\begin{array}{c}\text { Production(en millions de } \\
\text { tonnes ou d'hectolitres) }\end{array}$ & Rang mondial & Rang dans l'UE \\
\hline Vin & 43 & 2 & 2 \\
\hline Blé & 39 & 5 & 1 \\
\hline Lait & 24 & 5 & 2 \\
\hline Viande porcine & 2.3 & 6 & 3 \\
\hline Maïs & 16 & 7 & 1 \\
\hline Sucre & 4.5 & 8 & 1 \\
\hline Viande bovine & 1.8 & 8 & 1 \\
\hline
\end{tabular}

Source: Agreste, 2009

Sur les campagnes de récoltes récentes, la France participe au marché international des céréales de façon stable (cf. figure ${ }^{\circ} 4$ ). Comme dans les autres pays, la production française de céréales est tirée par l'accroissement des besoins en alimentation animale. Les points forts des céréales françaises tiennent à leurs rendements élevés et réguliers, ainsi qu’à leur accès privilégié aux marchés (bonne organisation des filières, faibles distances, qualité du système logistique). Cependant, si la France conserve des coûts de production compétitifs pour le blé face aux producteurs d'Amérique du Nord, ce n'est en revanche pas le cas face aux autres pays exportateurs, pas plus que pour les autres productions, telle le maïs.

Figure $n^{\circ} 4$ : La récolte française de céréales

\begin{tabular}{|l|l|l|l|}
\hline & Surface (hectares) & Rendements (quintaux/hectares) & Production (tonnes) \\
\hline Blé Tendre & & & \\
$2012 / 13$ & 4868021 & 74 & 36174189 \\
$2011 / 12$ & 4985153 & 68 & 33976548 \\
Evol. En \% & $-2,35 \%$ & $8,73 \%$ & $6,17 \%$ \\
\hline Orge & & & \\
$2012 / 13$ & 1679195 & 68 & 11479166 \\
$2011 / 12$ & 1546875 & 57 & 811076 \\
Evol. En \% & $8,55 \%$ & $20,01 \%$ & $30,28 \%$ \\
\hline Maïs & & & \\
$2012 / 13$ & 1653244 & 92 & 15174743 \\
$2011 / 12$ & 1557328 & 100 & 15594820 \\
Evol. En $\%$ & $6,16 \%$ & $-8,34 \%$ & $-2,69 \%$ \\
\hline
\end{tabular}

Source: France AgriMer, Conseil spécialisé céréales, septembre 2012 
La figure 3 fait aussi apparaitre les performances moindres des productions animales, tant à l'échelle mondiale qu'européenne. En effet, dans le contexte actuel de la négociation sur la libéralisation des échanges internationaux à l'OMC (cycle de Doha) et dans la perspective de l'évolution de la PAC en 2013, l'avenir des productions animales et des industries agroalimentaires françaises associées est empreint d'incertitudes. On sait également que dans le nord de l'Europe, les principaux groupes d'abattage sont trois à quatre fois plus grands que les premiers français et ils ont délibérément choisi une stratégie internationale, certes commerciale, mais aussi de déploiement industriel et financier.

En raison de choix de politiques agricoles et commerciales, mais aussi des stratégies d'entreprises, les filières animales françaises sont donc de plus en plus directement confrontées à la compétition internationale, au sein de l'Union européenne ou face au reste du monde, ce qui conduit à une forte diminution du nombre d'éleveurs en France avec un accroissement du nombre d'exploitations de grande taille, soit intensives (production laitière, production porcine, engraissement de jeunes bovins), soit extensive (naissage dans les systèmes bovins allaitants).

L'exportation de la production française est tirée par le blé et le vin et les produits alcoolisés (figure $\mathrm{n}^{\circ}$ ).

Figure $\mathrm{n}^{\circ} 5$ : Les principales exportations françaises en 2010, en valeur.

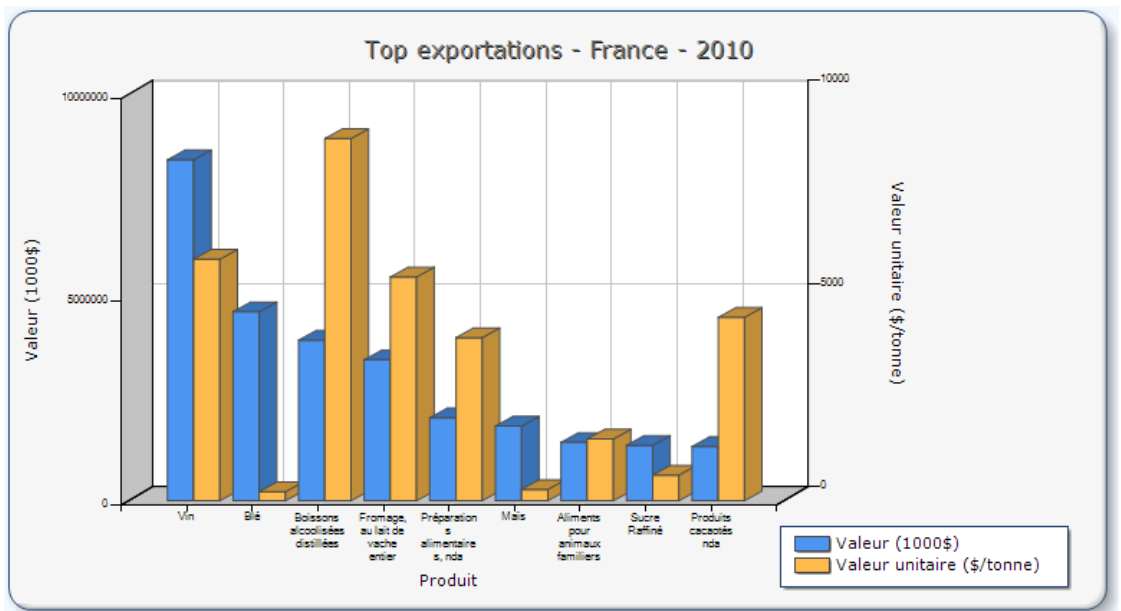

Source: FAO

Face aux difficultés d'approvisionnement qui s'annoncent sur le marché mondial des céréales, l'abondance des volumes français est un élément positif, à la fois pour le marché intérieur et pour les marchés d'exportation. La principale difficulté sera sans 
doute, comme en 2010/11, de trouver un équilibre entre la satisfaction des besoins intérieurs, d'une part, et du marché mondial, d'autre part. Ce dernier ne manquera pas, surtout si la Russie devait être peu présente à l'exportation, de se tourner vers l'origine française. De ce point de vue, la relative faiblesse de l'euro par rapport au dollar est un facteur de compétitivité à l'exportation, qui va rendre l'origine française d'autant plus convoitée.

Pour ce qui est du vin, la France est en 2011 le premier producteur de vin dans le monde, avec 51,1 millions d'hectolitres, mais le troisième pays exportateur. Les exportations mondiales, qui ont doublé en trente ans, représentent près de $43 \%$ de la consommation mondiale. C'est dire l'enjeu que représente le marché international. Les échanges sur le marché mondial des vins (les pays suivis pèsent près de $93 \%$ des échanges mondiaux) progressent régulièrement. Parties de moins de 50 millions d'hectolitres annuels dans les années 1980, les exportations atteindraient 103,5 millions d'hectolitres en 2011 soit près de $43 \%$ de la consommation mondiale et une hausse de près de $8 \%$ par rapport à 2010 . Le marché international représente un enjeu primordial pour tout pays producteur ayant une politique de développement de sa filière vitivinicole. Les exportations permettent non seulement de palier la faiblesse d'un marché local mais également de compenser le recul du débouché intérieur. En 2011, ce développement des échanges semble profiter aux pays traditionnellement exportateurs alors que depuis plusieurs années, cet essor bénéficie essentiellement aux pays du «Nouveau Monde». Ces derniers disposant généralement d'un faible marché intérieur, ce qui n'est pas le cas de la France. En 2011, la croissance des exportations s'est réalisée une nouvelle fois essentiellement à destination des pays tiers. En 2011, la croissance des exportations s'est donc faite une nouvelle fois essentiellement à destination des pays tiers.

Si la compétitivité de la France s'apprécie en premier lieu par sa performance à l'exportation sur les marchés mondiaux, cette approche mérite d'être complétée par la présentation de l'évolution des Investissements directs étrangers (IDE) entrants et sortants dans le secteur des IAA. Celle-ci constitue en effet un reflet de l'attractivité de la France pour cette industrie. Cette attractivité de la France peut être mesurée par les données d'IDE de la Banque de France (cf. figures nº et 7). Entre 2000 et 2008, l'évolution des IDE entrants et sortants dans le secteur des IAA a été selon la Banque de France plus dynamique que dans l'ensemble de l'économie, en particulier pour les IDE entrants, ce qui dénote une attractivité certaine de la France pour les investisseurs et correspond à l'approfondissement progressif du marché unique, la majorité de ces flux s'effectuant au sein de l'Union européenne. 
Figure $n^{\circ} 6$ : stock d'IDE français à l'étranger dans les IAA (valeur comptable), en millions d'euros, en 2000 (bleu) et en 2008 (rouge)

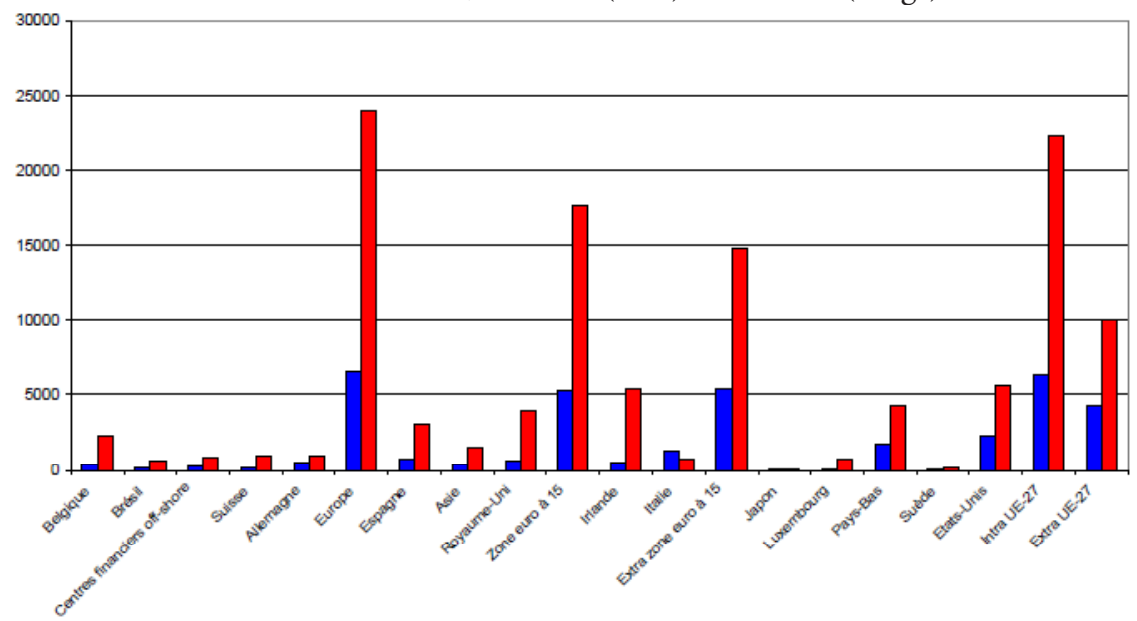

Source: Banque de France, CGEFI

Figure $n^{\circ} 7$ : stock d'IDE étrangers en France dans les IAA (valeur comptable)

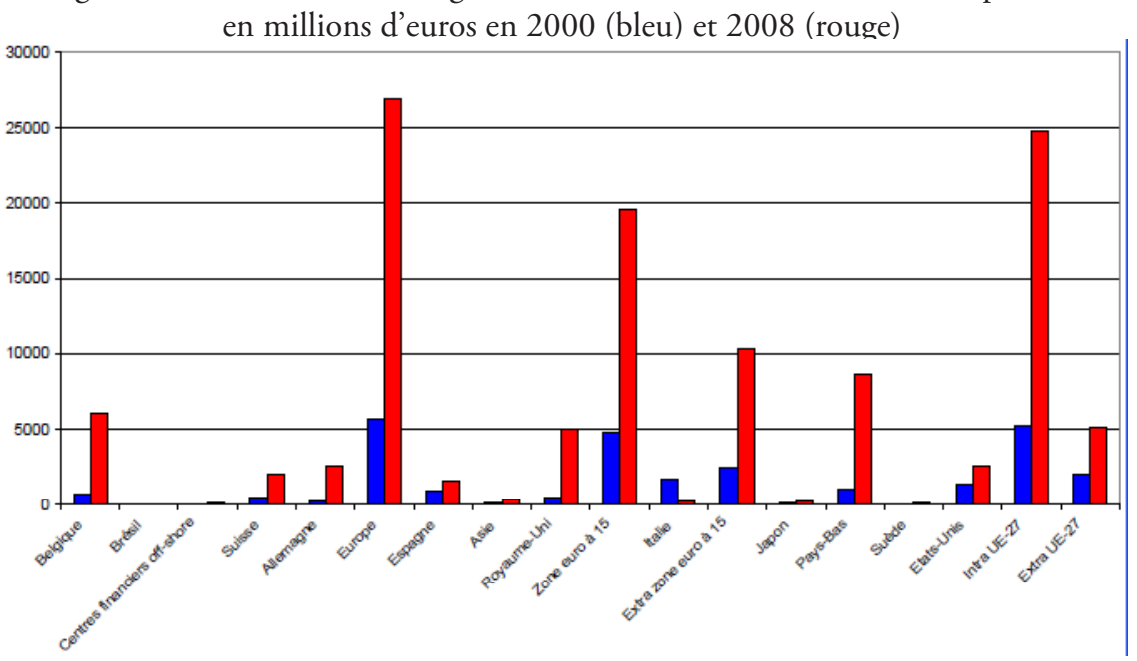

Source: Banque de France, CGEFI

Le stock des IDE en France a été multiplié par 4,1 et celui des IDE français à l'étranger (sortants) par 3. Le stock des IDE sortants reste un peu supérieur à celui des IDE en France. Cette évolution est plus dynamique que celle de l'ensemble 
des IDE, qui sont multipliés sur la même période par 2,5 pour les entrants et par 2 pour les sortants. Dans les deux cas, ces données suggèrent une attractivité certaine du site France, des entreprises françaises ou des marques françaises pour les investisseurs étrangers. La quasi-totalité des IDE entrants est le fait des pays de l'OCDE, et notamment de l'UE-27 (83\%). Les premiers investisseurs sont les PaysBas, la Belgique et le Royaume-Uni. Nos IDE s'effectuent à $92 \%$ dans les pays de l'OCDE et à $69 \%$ dans les pays de l'UE-27. Les stocks de nos IDE atteignent 1,5 Md€ en Asie et près d'1 Md€ en Amérique latine. Ces éléments semblent conforter l'idée de la constitution progressive du marché unique au sein de l'UE. Enfin, les statistiques publiées par la Banque de France montrent pour le secteur des IAA une forte progression du stock des IDE entrants et sortants depuis $2000^{9}$.

Au cours d'une période où se multiplient les évaluations quotidiennes de la compétitivité française - sans véritable effort de précision sur ce concept qui varie selon les écoles de pensée et le niveau d'analyse ${ }^{10}$ - il nous a paru opportun de présenter ces indicateurs qui n'ont pour but que de retracer la mesure des capacités et du travail accompli par le monde agricole. Il est évidemment bien plus complexe de mesurer les performances d'une activité telle que l'agriculture. Il s'agit à présent de voir les perspectives d'évolution et quelques grands enjeux pour l'agriculture française.

\section{Mieux Cerner le potentiel de l'agriculture française.}

\subsection{DES INCERTITUDES SUR L'AMÉLIORATION DES PERFORMANCES DES EXPLOITATIONS}

Les rendements céréaliers de la France ont fortement progressé depuis 20 ans, comme dans la plupart des régions agricoles. Les innovations technologiques et l'utilisation d'intrants sont les principaux facteurs explicatifs. Mais ils ne progressent quasiment plus et sont stables depuis les années 1990 (cf. figure $\mathrm{n}^{\circ} 8$ ).

9 Cf. Bruno Terrien, Stocks des investissements directs étrangers en France au 31 décembre 2009, Direction des enquêtes et statistiques sectorielles, Service des investissements directs, Banque de France; Lionel Fontagné, Farid Toubal, Investissement direct étranger et performances des entreprises, Conseil d'Analyse Économique, 2010.

10 Cf. Laure Latruffe, Johannes Sauer, Subsidies, Production Structure and Technical Change: A Cross-Country Comparison, 2010 Annual Meeting Agricultural and Applied Economics Association , 25-27 juillet 2010, Denver (Colorado). 
Figure $n^{\circ} 8$ : Stagnation récente des rendements céréaliers

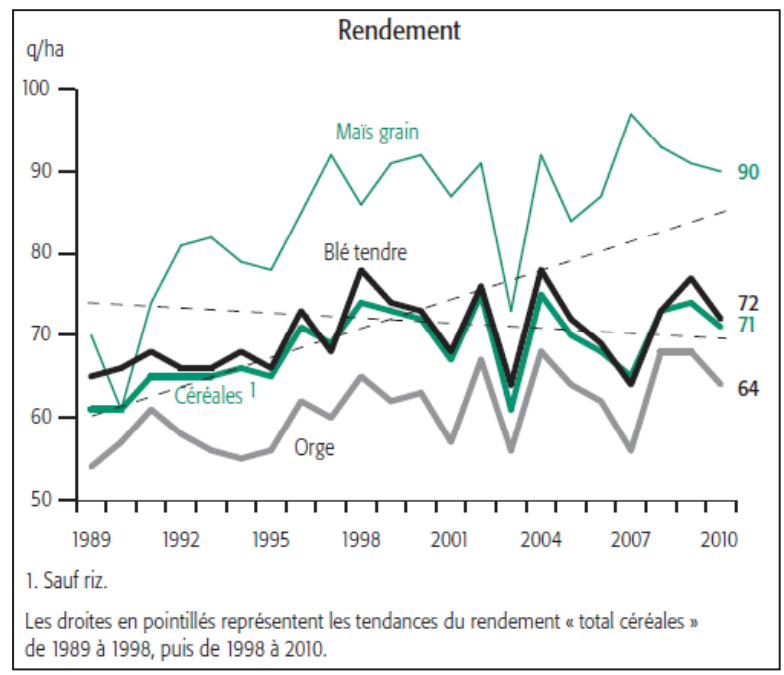

Source: SSP - Agreste - Statistique agricole annuelle

Les facteurs explicatifs sont potentiellement nombreux, qui ne s'excluent nullement: effets de la PAC (découplage des aides qui n'incite plus à la recherche systématique des gains de rendements), facteurs agronomiques (développement des parasites dans des systèmes de cultures simplifiés), facteurs climatiques (premiers effets du changement climatique), facteurs de pression sociopolitique environnementale, facteurs technologiques (recherche variétale qui marque le pas du fait du contexte économique peu propice aux variétés productives). Dans le futur, on peut autant compter sur la reprise de la hausse des rendements (si le contexte économique et technologique redevient favorable) que sur leur stagnation (des limites physiques et agronomiques pourraient être atteintes, notamment dans les systèmes de cultures très simples des grandes zones de production).

Outre la question des rendements et les réflexions quant aux itinéraires techniques, la libéralisation du secteur agricole confronte les acteurs de l'agriculture et des IAA aux prix mondiaux qui sont appelés à devenir l'instrument régulateur des dynamiques de production et d'échange à l'échelle européenne et mondiale. L'accroissement de leur volatilité depuis ces dernières années révèle d'une part une multiplication des sources de perturbation (effet des politiques énergétiques des pays développés, aléas de production, entrée en scène d'investisseurs financiers) et d'autre part une incertitude accrue sur les équilibres entre offre (état des stocks chinois par exemple) et demande (émergence d'importateurs massifs au Moyen-Orient entre autres). 


\subsection{DeUX PISTES POUR CERNER LE POTENTIEL DE CROISSANCE}

Il est désormais bien connu que l'exposition économique des agriculteurs au prix des intrants est forte. La nécessité de réduire les coûts de production constitue là un levier d'action possible pour accroître la productivité des exploitations. L'étude Ecophyto R\&D réalisée par l'INRA (Institut national de la recherche agronomique) montre qu'une optimisation des traitements permettrait une diminution de $15 \%$ des pesticides utilisés, sans changement de mode de production et sans changement du niveau de production. C'est une marge de manœuvre non négligeable pour les producteurs (et pour la protection de l'environnement).

L'énergie constitue également un facteur de compétitivité important. En effet, pour les exploitations agricoles le coût de l'énergie direct n'est pas négligeable. On constate d'ailleurs un développement des techniques simplifiées qui sont moins énergivores. L'évolution du prix de l'énergie, et du pétrole en particulier, impacte aussi fortement les exploitations agricoles via le prix des engrais minéraux. Enfin, la production d'énergie (cf. figure $\mathrm{n}^{\circ}$ ) sur l'exploitation peut être également source de compétitivité en dégageant une valeur ajoutée supplémentaire et en diversifiant les sources de revenu.

Figure $\mathrm{n}^{\circ} 9$ : Essor récent de la production d'agrocarburants

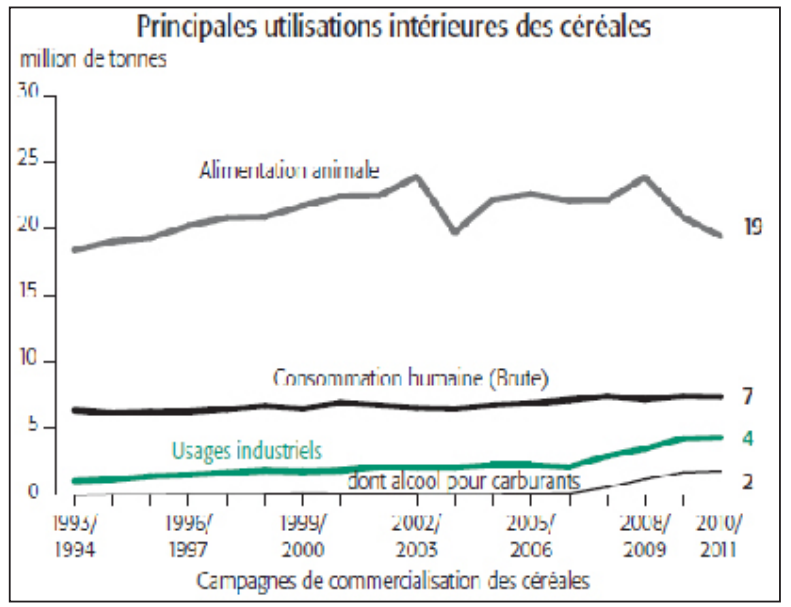

Source: SSP - Agreste - Statistique agricole annuelle

La diversification ou l'activité extra-agricole est envisagée de façon croissante comme une possibilité de renforcer les performances (prises au sens large) d'une exploitation. Elle connaît un léger regain. La vente directe et la transformation concernent davantage les petites exploitations que les grandes, surtout pour les activités de 
maraîchage, de viticulture et d'horticulture. Le tourisme à la ferme est plus répandu au sein des exploitations spécialisées en ovins, caprins et en arboriculture. On remarque également que les agriculteurs ayant diversifié les activités de leur exploitation travaillent avec une main- d'œuvre d'origine plus souvent familiale. Ils recourent davantage que les autres au salariat permanent. En 2000, leur niveau de formation était plus élevé que la moyenne. $57 \%$ de ceux qui avaient une activité touristique sur l'exploitation avaient au moins un niveau de formation secondaire long ou supérieur. La diversification peut continuer à prendre de l'importance. En effet, les Français tendent aujourd'hui à multiplier des courts séjours pendant leur temps libre et recherchent une proximité avec la nature qui peut favoriser l'agrotourisme. La vente directe peut bénéficier de la demande croissante des consommateurs.

\section{Conclusion}

Nous voudrions enfin mettre l'accent sur la ressource principale de l'agriculture française passée presque inaperçue dans cette présentation d'indicateurs et de tableaux: les hommes et les femmes qui produisent dans l'agriculture. En France, un exploitant sur quatre était âgé de plus de 55 ans en 2006, alors que les moins de 30 ans ne représentaient que $5 \%$ des agriculteurs ${ }^{11}$. Le renouvellement de la population agricole est mal assuré. Différents exercices de prévision existent qui tentent de modéliser le nombre futur d'agriculteurs à un horizon en général d'une dizaine d'années. Basés sur la prolongation des tendances actuelles, ils n'ont pas les mêmes objectifs et portent sur des périmètres différents (les chefs d'exploitations ou bien les exploitations elles-mêmes, ou plus largement l'ensemble de la famille professionnelle «Agriculteurs, sylviculteurs" par exemple). Ils s'accordent cependant sur la poursuite de la tendance à la baisse, à un rythme qui varie de 1,7 à 3,3\% par an selon les exercices ${ }^{12}$. À l'inverse, certains modes de production ou de valorisation semblent plus intensifs en emploi. Selon une publication récente de l'Inra ${ }^{13}$ sur l'arboriculture fruitière biologique, le passage à l'agriculture biologique génère du travail supplémentaire pour l'exploitant (3,5 unités de travail humain - permanents et salariés - pour 1000 tonnes produites en agriculture biologique contre 2,5 en agriculture conventionnelle) et peut être créateur d'emplois. Ces exercices mettent en évidence l'importance de deux facteurs: le nombre de départs à la retraite qui dépend des effectifs par classe d'âge

\footnotetext{
11 Cf. François Lefebvre, "Démographie agricole: la France doit-elle craindre I'avenir? Prévision à I'horizon 2020 ", Demeter 2009.

12 MSA, Le suivi statistique des cotisations des exploitants agricoles: outil de mesure du renouvellement des générations et des installations, confidentiel; Laurent Piet, Moins d'exploitations toujours plus grandes. La tendance va-t-elle se maintenir?, présen-tation au Salon international de l'agriculture; CAS-DARES, Les métiers en 2015, 2007; Institut de l'élevage, France laitière 2015. Vers une accentuation des contrastes régionaux, 2009; François Lefebvre, "Démographie agricole", op. cit.; Peter Nowicki, Vinetta Goba et al., Scenar 2020-II. Update of Analysis of Prospects in the Scenar 2020 Study, European Commission, Directorate-General Agriculture and Rural Development, Bruxelles, 2009.

13 Cf. Natacha Sautereau, Servane Penvern, Morgane Petitgenet, Joël Fauriel, Stéphane Bellon, "Concilier des performances pour une agriculture durable. L'agriculture biologique comme prototype ", FaçSADe. Résultats des recherches du département Inra-Sad (Sciences pour l'Action et le Développement), n²011/33, 4 p.
} 
ainsi que des mesures concernant la retraite (possibilité ou non de départ anticipé par exemple); l'évolution de la surface moyenne à l'installation et la disponibilité du foncier. Il s'agit d'une affaire de choix pour déterminer s'il faut encourager un transfert du foncier libéré vers de nouveaux entrants ou l'allouer à l'agrandissement. Le vieillissement de la population agricole et les disparités en termes de structures d'exploitation appellent à une réflexion sur les modèles d'exploitation qui coexistent aujourd'hui et qui devront trouver un équilibre entre performance, attractivité de la profession et durabilité.

Pour rester une grande puissance agricole, n'importe quel pays est donc contraint de mettre en ouvre une véritable politique de développement: la France est un exemple significatif avec les lois d'orientation agricoles des années 1960, puis avec la politique agricole commune. Cette politique ne consiste pas seulement, comme aux États-Unis et dans l'Union européenne, à fournir aux agriculteurs un minimum de garanties de prix ou de revenus ; elle consiste aussi, et peut-être même surtout, à mettre en œuvre une politique de formation et de recherche qui permette à l'agriculture concernée d'être durablement compétitive. À titre d'exemple, alors que la France avait la première agriculture biologique en Europe, en absolu et en proportion, au début des années $1980^{14}$, elle a accumulé depuis un retard conséquent qui semble se combler progressivement aujourd'hui.

14 Cf. Martial Saddier, L'agriculture biologique en France: vers la reconquête d'une première place européenne, Paris, La Documentation française, 2003, 335 p. 


\section{Annexe 1: Classement de la production française dans le monde par produit (2010, en valeur)}

\begin{tabular}{|c|c|}
\hline Position & Produit \\
\hline 1 & Orge \\
\hline 1 & Fibre et étoupe de lin \\
\hline 1 & Betterave à sucre \\
\hline 2 & Céréales mélangées \\
\hline 2 & Haricots verts \\
\hline 2 & Viande de canard indigène \\
\hline 3 & Fèves sèches \\
\hline 3 & Pois frais \\
\hline 3 & Viande de dinde indigène \\
\hline 4 & $\begin{array}{l}\text { Poireaux et autres légumes } \\
\text { alliacés }\end{array}$ \\
\hline 4 & Cillette \\
\hline 4 & Racines de chicorée \\
\hline 4 & Blé \\
\hline 4 & Graines de tournesol \\
\hline 5 & Choux-fleurs et brocolis \\
\hline 5 & Pois secs \\
\hline 5 & $\begin{array}{l}\text { Viande non définie } \\
\text { autrement (nda) }\end{array}$ \\
\hline 5 & Colza \\
\hline 5 & Raisins \\
\hline 5 & Kiwis \\
\hline 6 & Viande d'oie indigène \\
\hline 6 & Prunes et prunelles \\
\hline 6 & Lait de chèvre entier frais \\
\hline 7 & Lait de vache entier frais \\
\hline
\end{tabular}

\begin{tabular}{|c|c|}
\hline Position & Produit \\
\hline 7 & Maïs frais \\
\hline 7 & Pommes \\
\hline 7 & Viande de léporidés indigène \\
\hline 7 & Triticale \\
\hline 7 & Abricots \\
\hline 8 & Pommes de terre \\
\hline 8 & Moutarde \\
\hline 8 & Viande de bovins indigène \\
\hline 8 & Artichauts \\
\hline 8 & Laitue et chicorée \\
\hline 9 & Pêches et nectarines \\
\hline 9 & Étoupes Déchets de Chanvre \\
\hline 9 & Maïs \\
\hline 9 & Noix non décortiquées \\
\hline 9 & Noisettes non décortiquées \\
\hline 9 & Cassis et groseilles \\
\hline 10 & Eufs de poule en coquille \\
\hline 10 & Viande de suidés indigène \\
\hline 10 & Châtaignes \\
\hline 10 & Asperges \\
\hline 10 & Épinards \\
\hline 11 & Graines de lin \\
\hline 12 & Lait de brebis entier frais \\
\hline 12 & Cerises \\
\hline 12 & Houblon \\
\hline 12 & Avoine \\
\hline
\end{tabular}

Source: FAO 


\section{Annexe 2: Classement de la production française dans le monde par produit (2010, en quantité)}

\begin{tabular}{|c|c|}
\hline Position & Produit \\
\hline 1 & Son de céréales mélangées \\
\hline 1 & Amidons et fécules de blé \\
\hline 1 & Choux fourragers \\
\hline 1 & Betteraves fourragères \\
\hline 1 & Graisse de volaille \\
\hline 1 & Gluten de blé \\
\hline 1 & Farines de céréales mélangées \\
\hline 1 & Betterave à sucre \\
\hline 1 & Farine de moutarde \\
\hline 1 & $\begin{array}{l}\text { Aliments composés pour } \\
\text { volaille }\end{array}$ \\
\hline 1 & Sérum en poudre \\
\hline 1 & Huiles, graisses animales, nda \\
\hline 1 & Fibre et étoupe de lin \\
\hline 1 & $\begin{array}{l}\text { Babeurre, lait caillé, lait } \\
\text { acidifié }\end{array}$ \\
\hline 1 & Foie gras \\
\hline 1 & Graisses fondues de volaille \\
\hline 2 & $\begin{array}{l}\text { Sucre de betterave, brut, } \\
\text { centrifugé }\end{array}$ \\
\hline 2 & Chanvre \\
\hline 2 & Haricots verts \\
\hline 2 & Boissons alcoolisées distillées \\
\hline 2 & Ray-Grass Fourrager \\
\hline 2 & $\begin{array}{l}\text { Maïs doux préparé ou en } \\
\text { conserve }\end{array}$ \\
\hline 2 & Crème fraîche \\
\hline 2 & Fromage de lait de chèvre \\
\hline 2 & Viande de canard indigène \\
\hline 2 & $\begin{array}{l}\text { Viande de dinde } \\
\text { (Viand Din Bi) }\end{array}$ \\
\hline
\end{tabular}

\begin{tabular}{|c|c|}
\hline Position & Produit \\
\hline 2 & Marcs de raisins \\
\hline 2 & $\begin{array}{l}\text { Aliments composés pour } \\
\text { porcins }\end{array}$ \\
\hline 2 & Beurre de lait de vache \\
\hline 2 & $\begin{array}{l}\text { Viande de canard (Viand } \\
\text { Can Bi) }\end{array}$ \\
\hline 2 & Viand Can Bi \\
\hline 2 & Orge \\
\hline 2 & Glucose et dextrose \\
\hline 2 & Vin \\
\hline 2 & $\begin{array}{l}\text { Fromage, au lait de vache } \\
\text { entier }\end{array}$ \\
\hline 3 & Maïs fourrager \\
\hline 3 & $\begin{array}{l}\text { Oléagineux verts pour } \\
\text { l'ensilage }\end{array}$ \\
\hline 3 & Racines de chicorée \\
\hline 3 & Pois frais \\
\hline 3 & Yaourt concentré ou non \\
\hline 3 & Caséine \\
\hline 3 & Triticale \\
\hline 3 & Céréales mélangées \\
\hline 3 & Pois secs \\
\hline 3 & Préparations de foie \\
\hline 3 & Lait écrémé de vache \\
\hline 3 & Fèves sèches \\
\hline 3 & $\begin{array}{l}\text { Aliments composés pour } \\
\text { bovin }\end{array}$ \\
\hline 3 & Viande de dinde indigène \\
\hline 3 & $\begin{array}{l}\text { Fourrages et tourteaux au } \\
\text { gluten }\end{array}$ \\
\hline
\end{tabular}

Source: FAO 